\title{
Second and third harmonic generation from gold nanolayers: experiment versus theory
}

\author{
L. Rodríguez-Suné ${ }^{1,}$, J. Trull ${ }^{1}$, N. Akozbek ${ }^{2}$, D. De Ceglia ${ }^{3}$, M. A. Vincenti ${ }^{4}$, M. Scalora ${ }^{5}$, and C. Cojocaru ${ }^{1}$ \\ ${ }^{1}$ Department of Physics, Universitat Politècnica de Catalunya, Rambla Sant Nebridi 22, 08222 Terrassa, Spain \\ ${ }^{2}$ AEgis Technologies Inc., 401 Jan Davis Dr., Huntsville, Alabama 35806, USA \\ ${ }^{3}$ Department of Information Engineering - University of Padova, Via Gradenigo 6/a, 35131 Padova, Italy \\ ${ }^{4}$ Department of Information Engineering - University of Brescia, Via Branze 38, 25123 Brescia, Italy \\ ${ }^{5}$ Aviation and Missile Center, US Army CCDC, Redsone Arsenal, AL 35898-5000 USA
}

\begin{abstract}
The use of semiconductors, metals, or ordinary dielectrics in the process of fabrication of nanodevices is at the front edge of nowadays technology, exploiting the properties of light propagation and localization at nanometric scale in new and surprising ways. Understanding accurately how light interacts with these materials at the nanoscale is crucial if one is to properly engineer nano-devices. When the nanoscale is reached, light-matter interactions display new phenomena where conventional approximations may not always be applicable and they should be either revised or voided. In this work, we measure the efficiency of second and third harmonic generation from gold nanolayers. The experimental results are compared with numerical simulations based on a detailed microscopic hydrodynamic model that considers different effects playing a role in the nonlinear response, not usually considered by more generic models. The agreement between experimental and theoretical results proves the importance of all these contributions.
\end{abstract}

\section{Introduction}

More effort to properly engineer nano-antenas, filters, and other devices whose geometrical features approach atomic size, has been put in the recent years. For this reason, it is very important to understand how light interacts at the nanoscale with metals, semiconductors, or ordinary dielectrics. At this scale, light-matter interaction can display completely new phenomena and conventional approximations should be revised. This is the case, for instance, of two well-known nonlinear processes such as second and third harmonic generation (SHG and THG). These phenomena have been studied extensively in different optical materials. Usually, high conversion efficiencies are sought, what requires thick nonlinear materials with high nonlinearities, phase-matching conditions and low material absorption. Under these circumstances, the leading nonlinear polarization term corresponds to the bulk contribution described through the second and third order nonlinear susceptibility tensors $\left(\chi^{(2)}\right.$ and $\left.\chi^{(3)}\right)$. However, when the nanometric size is reached, $\mathrm{SH}$ and $\mathrm{TH}$ efficiencies may decrease and phase matching conditions and absorption may no longer play a primary or significant role. Moreover, the effective $\chi^{(2)}$ and $\chi^{(3)}$ may not coincide with their bulk, local counterparts and may depend on the type of nonlinearities that are triggered. In addition, contributions to the nonlinear polarization arising from electric quadrupole- like and magnetic sources should also be taken into account.

Most of the models used to explain harmonic generation from metals at the nanoscale rely on assigning effective surface and volume $\chi^{(2)}$ for SHG, and an effective volume $\chi^{(3)}$ to describe THG, which generally lack a detailed, microscopic, dynamical description of light propagation and light-matter interactions. With this, most theoretical predictions appear to accurately describe the general shape of the angular dependence of the SH signal, but fail in describing the observed amplitude.

In this work, we report experimental measurements on SHG and THG from $20 \mathrm{~nm}$ and $70 \mathrm{~nm}$-thick $\mathrm{Au}$ nanolayers. These measurements are compared with numerical simulations based on a microscopic hydrodynamic model which accounts for surface, magnetic and bulk nonlinearities arising from free and bound charges, preserving linear and nonlinear dispersion, nonlocal effects due to pressure and viscosity, and an intensity dependent free electron density. This model is adapted and applied anew based on previous work reported in references [1-4].

Corresponding author: laura.rodriguez.sune@upc.edu 


\section{Results}

\subsection{Experiments}

Measurements of SH signals from the 20nm-thick $\mathrm{Au}$ nanolayer have been conducted in transmission and in reflection while the SHG from the 70nm-thick $\mathrm{Au}$ nanolayers has been studied in reflection. In both cases, incident pulses tuned at $800 \mathrm{~nm}$ and $1064 \mathrm{~nm}$ have been used. The process of THG from the $20 \mathrm{~nm}$-thick layer was conducted either in transmission and reflection using incident pulses tuned at $1064 \mathrm{~nm}$. For this purpose, we have developed an experimental set-up, shown in Fig. 1, capable of analysing the angular dependence of the harmonic signals in both transmission and reflection configurations. First of all, a half-wave plate is used to control the polarization of the incident field. Then, we filter out any possible SH or TH coming from different optical components placed before the sample. We use a lens to focus the beam on the sample plane. A focal length $\mathrm{f}=200 \mathrm{~mm}$ was used for the SHG experiments to obtain beam intensities in the range of $1-2 \mathrm{GW} / \mathrm{cm}^{2}$. A focal length $f=100 \mathrm{~mm}$ was employed for the THG measurements in order to achieve higher beam intensities. The sample is mounted on a rotary support which allows us to take measurements as a function of the angle of incidence. Just after the sample, the fundamental field is attenuated by means of a filter in order to avoid any potential SHG or THG from the surfaces of the optical elements placed along the set-up after the sample. After that, a lens with focal length $\mathrm{f}=100 \mathrm{~mm}$ is used to collimate the beam, and a polarizer helps us to select the $\mathrm{SH}$ or $\mathrm{TH}$ polarization. Then, we use a prism and a blocking edge to separate and obscure the remaining fundamental field radiation from the $\mathrm{SH}$ or $\mathrm{TH}$ path. Finally, the harmonic signals are detected by means of a photomultiplier tube, on which we place a narrow-band spectral filter having $20 \mathrm{~nm}$ band pass transmission around either the $\mathrm{SH}$ or the
TH wavelength. This hole set-up is mounted on a rotary platform which allows us to take measurements in transmission and in reflection.

A calibration procedure has been performed to estimate the efficiency of a given process as the ratio between the SH or TH intensity generated in transmission or reflection, and the total peak pump pulse intensity just before the sample.

\subsection{Theory}

To understand the experimental results, we have performed numerical simulations reproducing the experimental situation. These simulations are based on a theoretical model that embraces full-scale, time-domain coupling of matter to the macroscopic Maxwell's equations. Our approach consists in formulating a microscopic, hydrodynamic model to understand linear and nonlinear optical properties of metals by accounting for competing surface, magnetic and bulk nonlinearities arising from both free and bound electrons, preserving linear and nonlinear material dispersion, nonlocal effects due to pressure and viscosity, and an intensity dependent free electron density to which we refer as hot electron contribution. When applying Newtonian dynamics to free and bound electrons, we obtain the simultaneous material equations of motion Eq. 1 and 2. Here, $\mathbf{P}_{\mathrm{b}, \mathrm{j}}$ and $\mathbf{P}_{\mathrm{f}}$ are bound and free electron polarizations, respectively. Eq. 1 describes the behaviour of bound electrons. The $\mathrm{j}$ counter indicates multiple bound electron spices. In a metal like $\mathrm{Au}$, one free (Drude) and two bound (Lorentz) electron spices generally suffice to describe the local, linear dielectric function down to a wavelength of $\sim 200 \mathrm{~nm}$. Each Lorentz spices is characterized by a third order, isotropic nonlinearity $\mathbf{P}_{\mathrm{b}, \mathrm{j}}^{\mathrm{NL}}=-\tilde{\beta}\left(\mathbf{P}_{\mathrm{b}, \mathrm{j}} \cdot \mathbf{P}_{\mathrm{bj}}\right) \mathbf{P}_{\mathrm{bj}}$, where the coefficient $\tilde{\beta}$ may be derived for a nonlinear, twodimensional oscillator model, and taking into account typical bound electron densities, lattice constant and

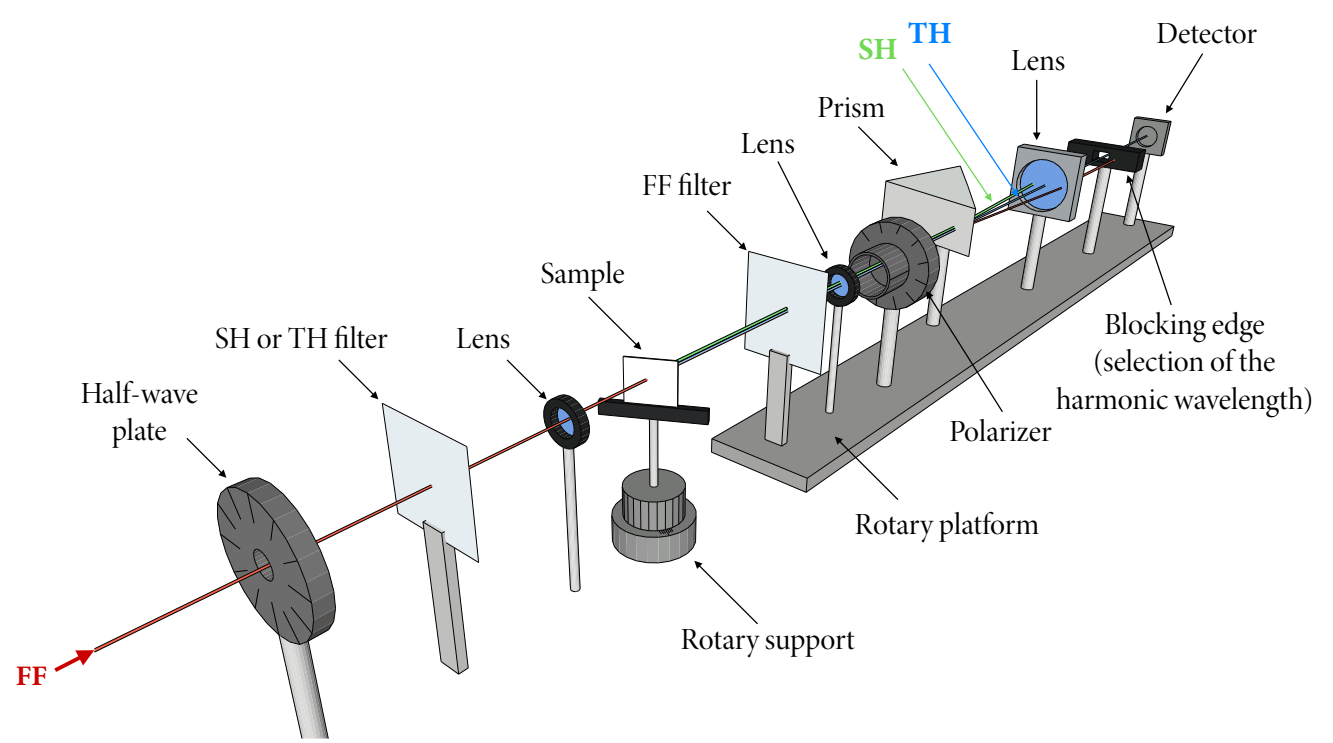

Fig. 1. Experimental set-up developed for measuring SH and TH signals from 20nm and 70nm-thick Au nanolayers. The rotary support allows us to rotate the sample so that we can take measurements as a function of the angle of incidence. The detection system is mounted on a rotary platform so that measurements can be taken in transmission and in reflection. 


$$
\begin{gathered}
\ddot{\mathbf{P}}_{\mathrm{b}, \mathrm{j}}+\tilde{\gamma}_{\mathrm{b}, \mathrm{j}} \dot{\mathbf{P}}_{\mathrm{b}, \mathrm{j}}+\widetilde{\omega}_{0, \mathrm{~b}, \mathrm{j}} \mathbf{P}_{\mathrm{b}, \mathrm{j}}+\mathbf{P}_{\mathrm{b}, \mathrm{j}}^{\mathrm{NL}}=\frac{\mathrm{n}_{0, \mathrm{~b}} \mathrm{e}^{2} \lambda_{0}^{2}}{\mathrm{~m}_{\mathrm{b}}^{*} \mathrm{c}^{2}} \mathbf{E}+\frac{\mathrm{e} \lambda_{0}}{\mathrm{~m}_{\mathrm{b}}^{*} \mathrm{c}^{2}} \mathbf{E}\left(\nabla \cdot \mathbf{P}_{\mathrm{b}, \mathrm{j}}\right)+\frac{\mathrm{e} \lambda_{0}}{\mathrm{~m}_{\mathrm{b}}^{*} \mathrm{c}^{2}} \dot{\mathbf{P}}_{\mathrm{b}, \mathrm{j}} \times \mathbf{H} \\
\ddot{\mathbf{P}}_{\mathrm{f}}+\tilde{\gamma}_{\mathrm{f}} \dot{\mathbf{P}}_{\mathrm{f}}=\frac{\mathrm{n}_{0, \mathrm{f}} \mathrm{e}^{2} \lambda_{0}^{2}}{\mathrm{~m}_{0, \mathrm{f}}^{*} \mathrm{c}^{2}} \mathbf{E}-\frac{\mathrm{e} \lambda_{0}}{\mathrm{~m}_{0, \mathrm{f}}^{*} \mathrm{c}^{2}} \mathbf{E}\left(\nabla \cdot \mathbf{P}_{\mathrm{f}}\right)+\widetilde{\Lambda}(\mathbf{E} \cdot \mathbf{E}) \mathbf{E}+\frac{\mathrm{e} \lambda_{0}}{\mathrm{~m}_{0, \mathrm{f}}^{*} \mathrm{c}^{2}} \dot{\mathbf{P}}_{\mathrm{f}} \times \mathbf{H}+\frac{3 \mathrm{E}_{\mathrm{F}}}{5 \mathrm{~m}_{0, \mathrm{f}}^{*} \mathrm{c}^{2}}\left(\nabla\left(\nabla \cdot \mathbf{P}_{\mathrm{f}}\right)+\frac{1}{2} \nabla^{2} \mathbf{P}_{\mathrm{f}}\right)+ \\
-\frac{1}{\mathrm{n}_{0, \mathrm{f}} \lambda_{0}}\left[\left(\nabla \cdot \dot{\mathbf{P}}_{\mathrm{f}}\right) \dot{\mathbf{P}}_{\mathrm{f}}+\left(\dot{\mathbf{P}}_{\mathrm{f}} \cdot \nabla\right) \dot{\mathbf{P}}_{\mathrm{f}}\right]
\end{gathered}
$$

resonance frequencies for this material, it has a value of $\tilde{\beta} \approx 10^{-8}$. This parameter governs all third order effects triggered by the background crystal, i.e. bound electrons, including self-phase modulation, nonlinear absorption, and THG power conversion efficiencies. Eq. 2 determines the action of free electrons. As it can be seen, apart from the usual linear driving term there are different free electron contributions that give rise to harmonic generation. A quadrupole-like Coulomb term $-\frac{\mathrm{e} \lambda_{0}}{\mathrm{~m}_{0, \mathrm{f}}^{*} \mathrm{c}^{2}} \mathbf{E}\left(\nabla \cdot \mathbf{P}_{\mathrm{f}}\right)$, a magnetic Lorentz term $\frac{\mathrm{e} \lambda_{0}}{\mathrm{~m}_{0, \mathrm{f}}^{*} \mathrm{c}^{2}} \dot{\mathbf{P}}_{\mathrm{f}} \times \mathbf{H}$, convection terms $-\frac{1}{\mathrm{n}_{0, \mathrm{f}} \lambda_{0}}\left[\left(\nabla \cdot \dot{\mathbf{P}}_{\mathrm{f}}\right) \dot{\mathbf{P}}_{\mathrm{f}}+\left(\dot{\mathbf{P}}_{\mathrm{f}} \cdot \nabla\right) \dot{\mathbf{P}}_{\mathrm{f}}\right]$, nonlocal effects represented by pressure and viscosity $\frac{3 \mathrm{E}_{\mathrm{F}}}{5 \mathrm{~m}_{0, \mathrm{f}}^{*} \mathrm{c}^{2}}\left(\nabla\left(\nabla \cdot \mathbf{P}_{\mathrm{f}}\right)+\frac{1}{2} \nabla^{2} \mathbf{P}_{\mathrm{f}}\right)$, and the hot electrons contribution $\widetilde{\Lambda}(\mathbf{E} \cdot \mathbf{E}) \mathbf{E}$. In this work, we highlight and discuss for the first time the relative roles bound and hot electrons play in THG and end up with the conclusion that the generated TH signal is mostly triggered by hot electron dynamics.

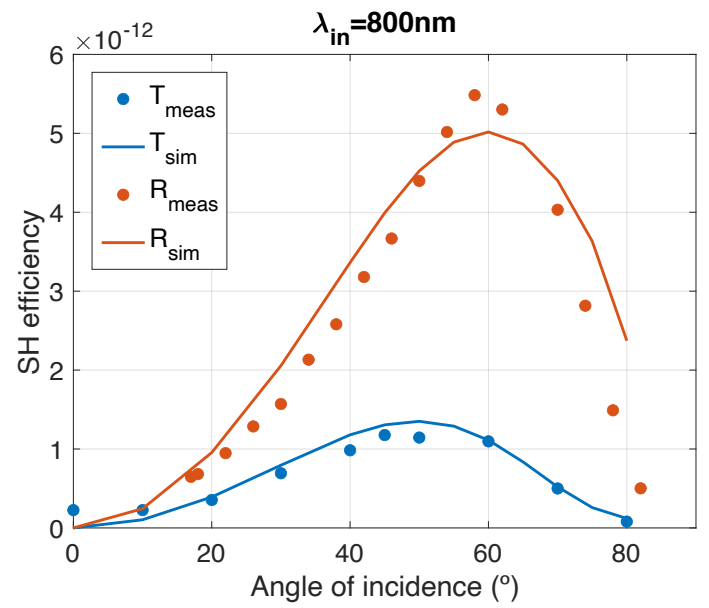

Fig. 2. Measured (full circles) and predicted (solid curves) SHG efficiencies as a function of the angle of incidence from the $20 \mathrm{~nm}$-thick Au layer when the incident pulse is tuned at $800 \mathrm{~nm}$. Transmission and reflection are plotted in blue and red, respectively.

In this section, we present some of the experimental results that we have obtained, which are compared with their corresponding numerical predictions.

In Fig. 2 we show results of SHG from the $20 \mathrm{~nm}$-thick Au layer when the sample is illuminated at $800 \mathrm{~nm}$. Experiments are depicted using full circles and simulations using solid curves. Transmitted (blue) and reflected (red) efficiencies are plotted as a function of the angle of incidence. As it can be seen, the maximum of the reflected curve is a little bit shifted towards a larger angle of incidence with respect to the transmitted curve. This phenomenon can be appreciated in both experimental and predicted results. Moreover, the ratio between the measured reflected and transmitted efficiencies is also reproduced in the theoretical case.

In Fig. 3 we present the reflection measurements (full circles) of the SH signal generated by the 70nm-thick $\mathrm{Au}$ layer when it is illuminated at $1064 \mathrm{~nm}$. As it can be seen, the maximum of the curve is achieved at a large angle of incidence, around $70^{\circ}$, fact that is reproduced in the predicted results.

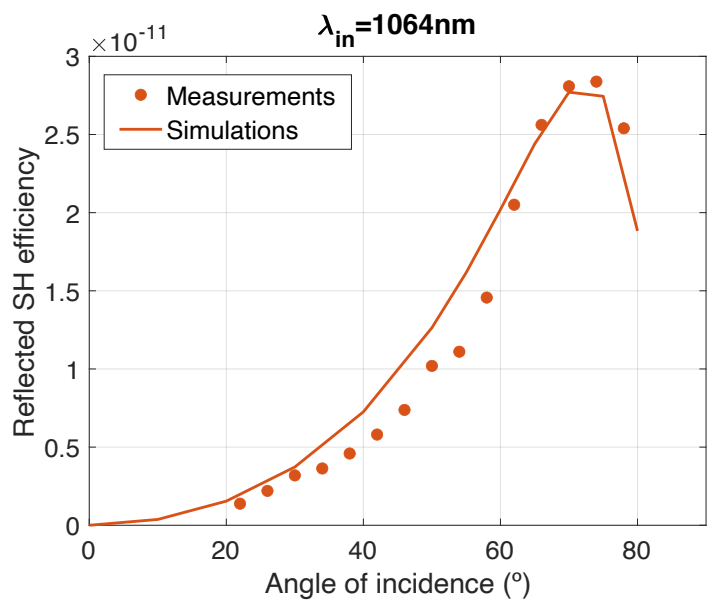

Fig. 3. Measured (full circles) and predicted (solid curves) SHG transmitted efficiencies as a function of the angle of incidence from the 70nm-thick Au layer when the incident pulse is tuned at $1064 \mathrm{~nm}$.

In Fig. 4, we show the results of the transmitted TH from the $20 \mathrm{~nm}$-thick Au layer as a function of the angle of incidence when the input pulse is tuned at $1064 \mathrm{~nm}$. In blue, we plot the transmitted TM-polarized TH for a TMpolarized incident pump, and in red we represent the transmitted TE-polarized TH generated when the incident field is TE-polarized. The simulations are performed by taking both bound and hot electrons into account. By only introducing the bound electrons contribution, efficiencies of order $10^{-11}$ where obtained, clearly inadequate to explain our measurements. Instead, what is required to reproduce the conversion efficiencies that we observe is the introduction of the hot electrons contribution.

In order to see how important the bound and hot electrons contribution to the TH signal can be, we have plotted in Fig. 4 predictions of the reflected TH efficiency from a $20 \mathrm{~nm}$-thick Au layer as a function of the input wavelength for three different scenarios: only taking bound electrons into account (black), only taking hot electrons into account (red) and taking both contributions into account (blue). It can be seen that, separately, each type of third order nonlinearity yields quantitavely and qualitavely a 
similar response, with a TH peak for pump wavelength of $\sim 600 \mathrm{~nm}$. However, their combined response redshifts the $\mathrm{TH}$ peak. This prediction runs counter to intuition because an increased free electron density should blueshift the plasma frequency, with expectedly similar outcome for the TH peak. It is obvious that the two components interfere and conspire to instead redshift the peak, an effect that encapsulated a cautionary tale for any experimental result that may be cavalierly extrapolated without the benefit of proper assumptions and theoretical support.

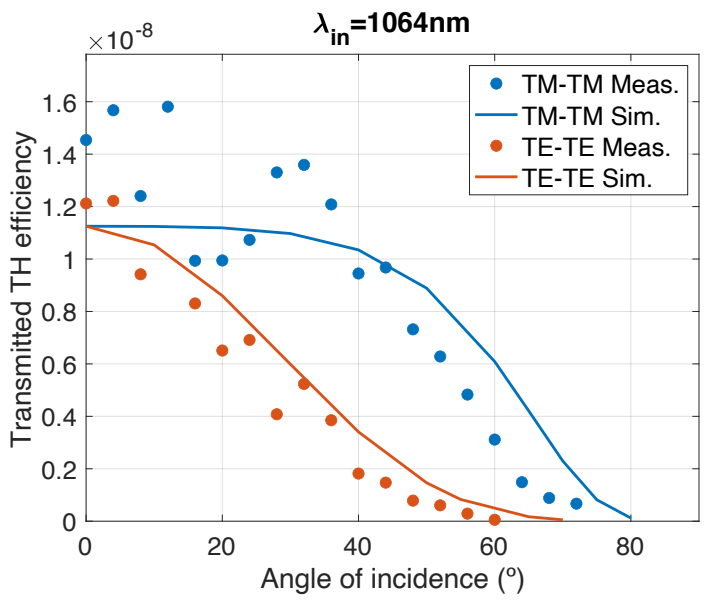

Fig. 4. Measured (full circles) and predicted (solid curves) transmitted THG efficiencies as a function of the angle of incidence from the 20nm-thick Au layer when the incident field is tuned at $1064 \mathrm{~nm}$. A TM- (blue) and TE-polarized (red) TH field is detected when the fundamental field is TM- or TEpolarized, respectively.

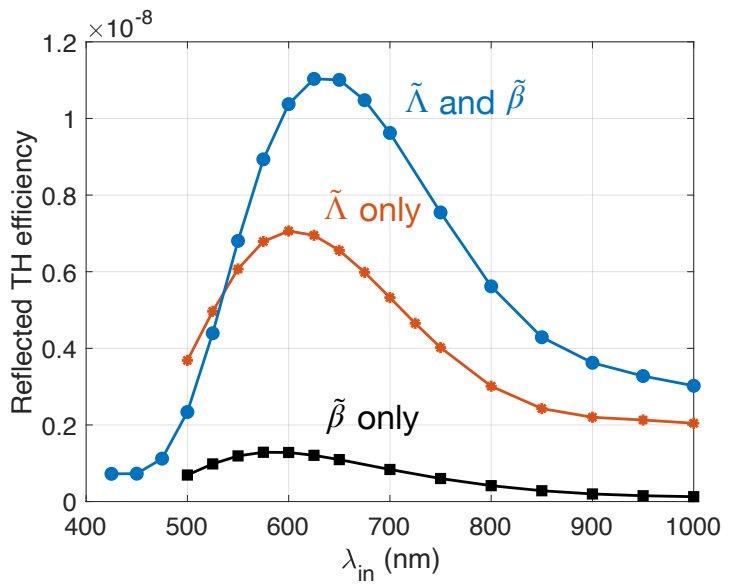

Fig. 5. Predictions of the reflected TH efficiency as a function of the pump wavelength obtained under three different scenarios: taking only bound electrons into account (black), takin only hot electrons into account (red), and taking both bound and hot electrons into account (blue).

\section{Conclusions}

In conclusion, we have reported measurements on SHG and THG from $20 \mathrm{~nm}$ and $70 \mathrm{~nm}$-thick Au layers. The SH field generated by the $20 \mathrm{~nm}$-thick sample measured as a function of the angle of incidence carries information about combined surface and volume currents excited on and inside the sample. The angular dependence of the reflected SH signal from the 70nm-thick $\mathrm{Au}$ layer supports mostly surface currents. These measurements are compared with numerical simulations based on a hydrodynamic approach to model light-matter interactions that make no assumptions about effective surface or volume nonlinearities. Instead, we rely on temporal and spatial derivatives and mere knowledge of the effective electron mass to determine the relative magnitudes of surface and volume contributions. With this approach we find remarkable agreement with experimental observations. The THG measurements and simulations are reported for TM and TE polarizations of the generated field from the $20 \mathrm{~nm}$-thick layer in transmission and as a function of the angle of incidence. The generated TH signal is attributed mostly to hot electron dynamics.

\section{References}

1. M. Scalora, M. A. Vincenti, D. de Ceglia, V. Roppo, M. Centini, N. Akozbek and M. J. Bloemer, Phys. Rev. A 82, 043828 (2010).

2. M. Scalora, M. A. Vincenti, D. de Ceglia, C. M. Cojocaru, M. Grande and J. W. Haus, J. Opt. Soc. Am. B 32, 2129-2138 (2010).

3. L. Rodríguez-Suné, M. Scalora, A. S. Johnson, C. Cojocaru, N. Akozbek, Z. J. Coppens, D. PerezSalinas, S. Wall and J. Trull, APL Photonics 5, 010801 (2020).

4. M. Scalora, J. Trull, D. de Ceglia, M. A. Vincenti, N. Akozbek, C. Coppens, L. Rodríguez-Suné and C. Cojocaru, Phys. Rev. A 101, 053828 (2020). 